\title{
Preparation and Stability of Nanofluids-A Review
}

\author{
Sayantan Mukherjee ${ }^{1}$, Somjit Paria ${ }^{1}$ \\ ${ }^{1}$ School of Mechanical Engineering, KIIT University, Bhubaneswar-751024, Odisha, India
}

\begin{abstract}
Nanofluid, a simple product of nanotechnology has become a topic of attraction due to its extraordinary heat transfer performance in various areas including cooling, power generation, defense, nuclear, space, microelectronics and biomedical appliances. However, preparation and stabilization of such fluids are indeed a matter of concern for better understanding. For the last decade numerous research and development works have been done in the synthesis and stability of such materials. In this contribution, a brief review has been presented to provide an update about the preparation and stabilization methods of nanofluids.
\end{abstract}

Keywords: Nanofluids, preparation, stability

\section{Introduction}

Nanofluids are colloidal suspension of ultra-fine metallic or nonmetallic particles in a given fluids. Despite all other properties, it is well known for its high thermal conductivity and better response as heat transfer medium. Nanofluids can be of two kinds such as metallic nanofluids and nonmetallic nanofluids. Metallic nanofluids are prepared by dispersing nanoparticle made from metals such as aluminum, copper, nickel etc. and nonmetallic nanofluids are made by dispersing nanoparticles of nonmetals i.e. metal oxides, various allotropes of carbon (Graphene, CNT) etc.Synthesis and stability of nanofluids are the two very primary requirements to study nanofluids. The proper utilization of the potential of nanofluids depends on the preparation and stability of nanofluids. The later part of this section itis intended to present these two very important terms.

\subsection{History and development of nanofluids}

The twenty first century is an era of technological development and has already seen many changes in almost every industry. The introduction of nanoscience and technology is based on the famous phrase "There's Plenty of Room at the bottom" by the Nobel Prize winning physicist Richard Feynman in 1959.Feynman proposed the concept of micromachines. In 1974 Scientist Norio Taniguchi first used the term 'Nanotechnology'. After that in 1995, scientist Choi of Argonne Laboratory (USA) successfully prepared nanofluid. After the establishment of such a landmark in the history of nanoscience, nanofluids grab the attention of the most of the researchers around the globe. In recent years the craze about nanofluid research has gone to such a hype that in the year 2011 alone, there were nearly700 research papers where the term nanofluid was used showing abreakneck growth from 2006(175) and 2001(10)[1].Figure1 shows an exponential growth in nanofluid research.

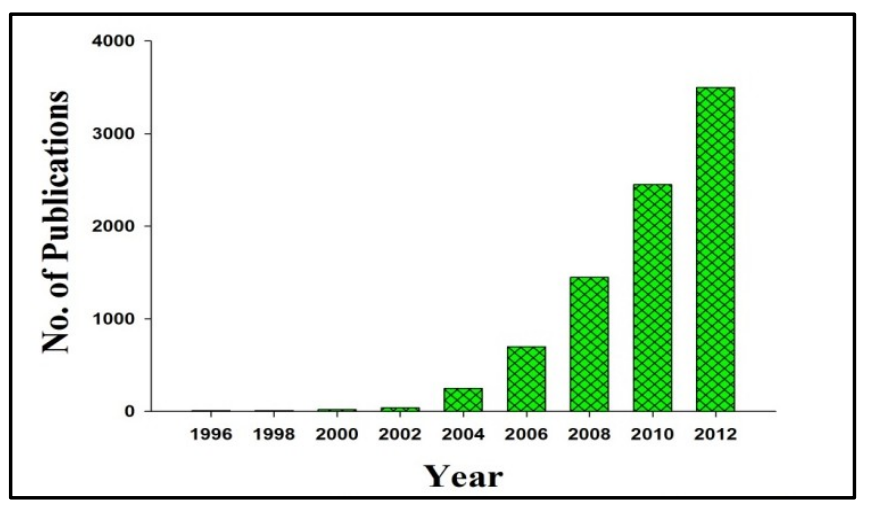

Figure1: Year wise publication on nanofluids

\subsection{Potential features of nanofluids}

Nanofluids have some uncommon features those make them very special for various engineering applications. Some of the special qualities of nanofluids are:

I. Rise in thermal conductivity beyond exception and much higher than theoretical predictions.

II. Ultrafast heat transfer ability. 
III. Better stability than other colloids.

IV. Reduction of erosion and clogging in micro channels.

V. Reduction in pumping power

VI. Reduce friction coefficient.

VII. Better lubrication.

\section{Preparation of nanofluids}

Preparation of nanofluids is the first foot-step to the experimental studies of nanofluids. There are two primary methods to prepare nanofluids: the single-step preparation process and the two-step preparation process. A brief discussion on nanofluid preparation processes is given in 2.1,2.2 and 2.3.

\subsection{Single-step preparation process}

The single-step preparation process indicates the synthesis of nanofluids in one-step. Several singlestep methods have been arrived for nanofluid preparation. Akoh et al. [2] developed a single-step direct evaporation method. This process is familiar as VEROS (Vacuum Evaporation onto a Running Oil Substrate).But it was difficult to separate nanoparticles form fluids. Eastman et al. [3] developed a modified VEROS technique, in which $\mathrm{Cu}$ vapor is directly condensed into nanoparticles by contact with flowing lowvapor-pressure ethylene glycol.Zhu et al.[4] presented a single-step chemical process for the preparation of $\mathrm{Cu}$ nanofluids by reducing $\mathrm{CuSO}_{4} .5 \mathrm{H}_{2} \mathrm{O}$ with $\mathrm{NaH}_{2} \mathrm{PO}_{2} . \mathrm{H}_{2} \mathrm{O}$ in ethylene glycol under microwave irradiation. This method also proved to be a good way to produce mineral oil based silver nanofluids.Lo et al. [5] developed vacuum based submerged arc nanoparticle synthesis to prepare $\mathrm{CuO}, \mathrm{Cu}_{2} \mathrm{O}$ and $\mathrm{Cu}$ based nanofluids with different dielectric liquids. A suitable power source is required to produce an electric arc between $6000-12000^{\circ} \mathrm{C}$ which melts and vaporizes a metal rod in the region where arc is created. The vaporized metal is condensed and then dispersed by deionized water to produce nanofluids. An advantage of one-step synthesis method is that nanoparticle agglomeration is minimized. But primeproblem is that only low vapor pressure fluids are compatible with such a process. One-step preparationprocess (chemical process) of nanofluids is given in the Figure2.

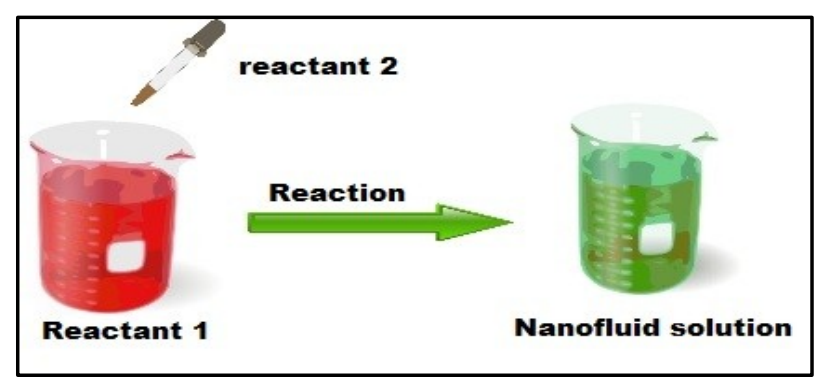

Figure2:One-step preparation process of nanofluids

\subsection{Two-step preparation process}

Two-step preparation process is extensively usedin the synthesis of nanofluids by mixing base fluids with commercially available nanopowders obtained from different mechanical, physical and chemical routes such as milling, grinding, and sol-gel and vapor phase methods. An ultrasonic vibrator or higher shear mixing device is generally used to stir nanopowders with host fluids. Frequentuseof ultrasonicationor stirring is required to reduce particle agglomeration. Eastman et al [3], Lee et al. [6], Wang et al. [7] used two-step method to produce alumina nanofluids. Murshed et al. [8] prepared $\mathrm{TiO}_{2}$-water nanosuspension by the same method. Xuan et al. [9] used commercially available $\mathrm{Cu}$ nanoparticles to prepare nanofluids of both water and transformer oil. Kim et al. [10] used two-step method to prepare $\mathrm{CuO}$ dispersed ethylene glycol nanofluids by Sonication and without stabilizers. Two-step method can also be used for synthesis of carbon nanotube based nanofluids. Single -walled and multi-walled carbon nanotubes are first produced by pyrolysis method and then suspended in base fluids with or without the use of surfactants $[30,40,41]$. Some authors suggested thattwo-step process is very suitable to preparenanofluids containing oxide nanoparticles than those containing metallic nanoparticles [11]. Stability is a big issue that inherently related to this operation as the powders easily aggregate due to strong van der Walls force among nanoparticles. In spite of such disadvantages this process is still popular as the most economic process for nanofluidsproduction. The most common two-step method is shown in Figure3. 


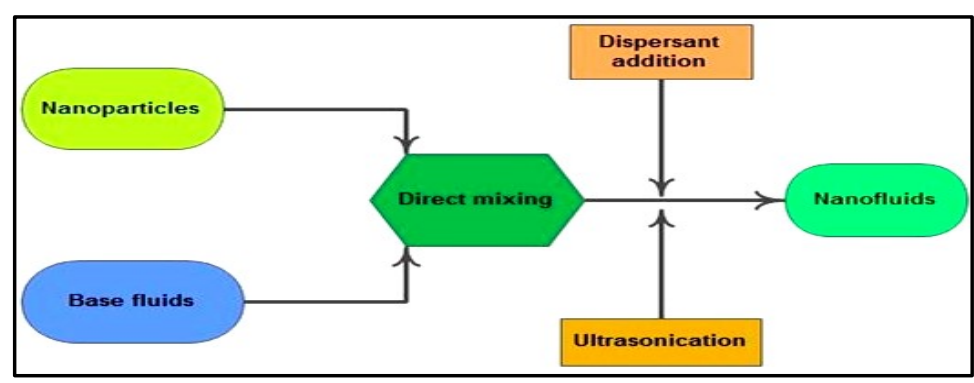

Figure3: Two step preparation process of nanofluids

\subsection{Other methods}

There are some other literatures where different approaches (other than these two procedures) were expressed. Feng et al. [12] usedaqueous organic phase transfer method for preparation of gold, silver, platinum nanoparticles. Phase transfer method can also be applied to prepare kerosene based $\mathrm{Fe}_{3} \mathrm{O}_{4}$ nanofluids which do not show time dependent thermal conductivity. Grafting of oleic acid onto the surface $\mathrm{Fe}_{3} \mathrm{O}_{4}$ makes it compatible with kerosene [13]. Wei et al. [14] established a continuous flow microfluidic microreactor to synthesize copper nanofluids. The microstructure and properties of nanofluids can appropriatelybe varied by adjusting parameters such as concentration, flow rate, additives. Moreover a novel preparation of aqueous $\mathrm{CuO}$ nanofluid can be done through novel precursor transformation method with the help of ultrasonic and microwave irradiation [15]. Here the precursor, $\mathrm{Cu}(\mathrm{OH})_{2}$ is completely converted to $\mathrm{CuO}$ in water under that process. The use of ammonium citrate is to prevent the growth and aggregation of nanoparticles, resulting in a stable $\mathrm{CuO}$ aqueous nanofluid with higher thermal conductivity than those prepared by other dispersion methods.

\section{Stability of nanofluids}

Nanofluids which can lose their potential to transfer heat due to their proneness to coagulation. Therefore, investigation on stability is an unavoidable issue that can alter the thermo-physical properties of nanofluids for application and also important to analyze the influential factors to the stability of such suspensions. This section contains the stability evolution methods and stability enhancement processes along with a detail about the stability mechanisms related to nanofluids.

\subsection{Stability evaluation methods for nanofluids}

\subsubsection{Zeta potential analysis}

Zeta potential is the potential difference between the dispersion medium and the stationary layer of fluid attached to the particle. The zeta potential indicates the degree of repulsion between adjacent, similarly charged particles in dispersion (Figure4).So, colloids with high zeta potential (negative or positive) are electrically stabilized while colloids with low zeta potentials tend to coagulate or flocculate. Nanofluids with zeta potential from $40-60 \mathrm{mV}$ are believed to have excellent stability. A lot of researchershave gone through zeta potential test of nanofluids. Kim et al. [16] used zeta potential analysis for Au nanofluids and found out standing stability. Zhu et al. [17] measured the zeta potential of Alumina-water based nanofluids under different $\mathrm{pH}$ values and different SDBS concentrations. The DLVO theory was applied to measure the attractive and repulsive potentials.

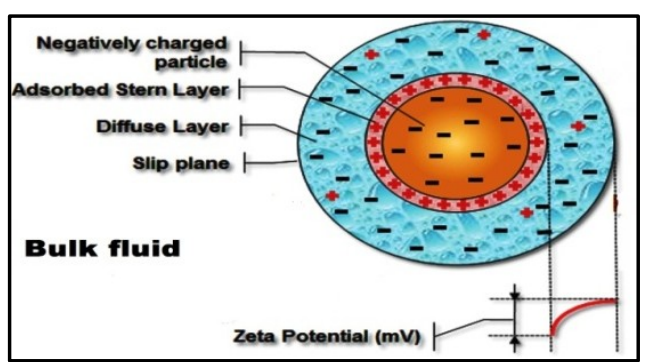

Figure4: Zeta potential of nanofluids

\subsubsection{Sedimentation method}

Sedimentation method is the most elementary method forevaluation of nanofluids [14]. An external force field is applied to start the sedimentation of nanoparticles in the nanofluids. The weight of sediment or the volume of sediment indicates the stability of nanofluids. Nanofluids are generally considered to be stable if the concentration of the supernatant particles remains constant with time.Zhu et al. [18] used the principle of 
sedimentation method in his own experimental setup to measure the stability of graphite suspension. Use of camera has proven to be a suitable aid to capture sedimentation photographs for observing the stability of nanofluids [36].Waiting time for capturingphotos links up with quality of nanofluids during preparation and well use of applied methods to make astable nanofluids. Wei et al. captured photographs of their samples within 24 hours after preparation. Wang et al. followed the path for testing sedimentation of alumina-water nanofluid [32].

\subsubsection{Centrifugation method}

Sedimentation method is very time consuming as it requires a long period of observation. So centrifugation method is developed for stability evaluation. Sing et al. [19] used centrifugation method to evaluate the stability of silver nanofluid prepared by reducing $\mathrm{AgNO}_{3}$ and selecting PVP as the stabilizer. An excellent stability of silver nanofluids was found due to the protective role of PVP because it decelerates the agglomeration of particles by steric effect.

\subsubsection{Spectral analysis method}

Spectral analysis via UV- vis spectrophotometer is another useful way to evaluate stability of nanofluids. The advantageover other methods that $\mathrm{UV}$-vis spectroscopy gives quantitative results corresponding to concentration of nanofluids. Hwang et al. [20] analyzed the stability of MWNT nanofluids by measuring the UV-vis absorption of MWNT at different sediment time.

The above three method can be used all together to complete the stability evaluation process. For example Li et al. [21] performed zeta potential analysis, absorbency and sedimentation photography for copper nanofluids under different $\mathrm{pH}$ values, different dispersion types and different concentrations.

\subsubsection{Method}

In this method, stability of suspensions can be evaluated considering thermal conductivity growth caused by the nanoparticle sedimentation in a wide nanoparticle volume fraction range [34]. A new literature has found using this method to check the stability of nanofluids [35].

\subsubsection{Electron microscopy and light scattering methods}

Measurement of particle size distribution by microscopy and light scattering techniques are two general methods for observing particle aggregation. Very high resolution microscope such as TEM and SEM are applied to capture the digital image ofnanoparticles, known as electron micrograph.Figure5 $(a, b)$ shows TEM and SEM photographs of CuOnanoparticles respectively [38, 39]. Cryogenic electron microscopy canbe used for the same purpose if themicrostructure of nanofluids is not changed during cryoation [37].Light scattering technique can also be used for the study of complex nanosuspensions.

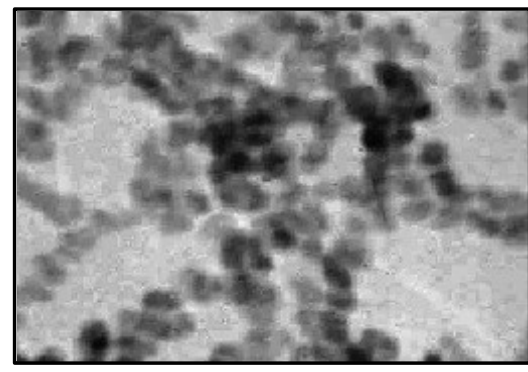

(a) (b)

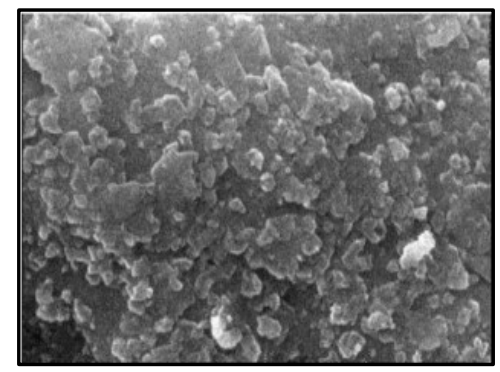

Figure5: a) TEM of $\mathrm{CuO}$ nanoparticles [38] b) SEM of CuO nanoparticles [39]

\subsection{Stability enhancement procedures \\ 3.2.1. Addition of surfactants}

Surfactants or dispersants are generally applied to stabilize the nanofluids. Addition of surfactants lowers the surface tension of host fluids and increases the immersion of particles.Surfactants can be defined as chemical compounds added to nanoparticles in order to lower surface tension of liquids and increase immersion of particles. Several literatures talk about adding surfactant to nanoparticles to avoid fast sedimentation; however, enough surfactant should be added to particle at any particular case. In researches, several types of surfactant had been utilized for different kinds of nanofluids. Some important surfactants are: Sodium dodecyl sulfate (SDS)[22], Salt and oleic acid [23], Dodecyltrimethylammonium bromide (DTAB) [24], Hexadecyltrimethylammoniumbromide (HCTAB) [13], Polyvinylpyrrolidone (PVP)[25],Gum Arabic [26]. It should be noted that this technique cannot be applicable for nanofluids working in high temperature on account of probable damage of bonding between surfactant and nanoparticle. Additionally surfactants may 
hamper heat transfer produce foam when heating. Furthermore surfactants may increase the thermal resistant between the nanoparticle and the base fluids which may lead diminish the enhancement in the thermal conductivity [27].

\subsubsection{Surface modification techniques}

This segment presents the surfactant free method. Injection of functional nanoparticles in the base fluids can provide long term stability of nanofluids. There are a number of examples of such modification techniques. As for example, Yang et al. [28] grafted silanes directly to the surface of silica nanoparticles in the original nanoparticle solutions. A special feature of those nanofluids was no deposition layer formed on the heated surface after a pool boiling process. The stability of carbon nanotubes can be increased by introducing hydroxyl groups onto the surface of CNTs[29].Plasma treatment can be applied to modify the surface of diamond nanoparticles for improving their dispersion property in water [30]. Detailsof surface modification techniques can be found in the reference [27].

\subsection{3. pH control of nanofluids}

Stability of nanofluid is directly related to its electro-kinetic properties; therefore, $\mathrm{pH}$ control of them can increase stability due to strong repulsive forces .As for example, simple acid treatment could cause nice stability of CNT in water[31].Lee et al.[32]investigated various $\mathrm{pH}$ values for $\mathrm{Al}_{2} \mathrm{O}_{3}$ nanofluid and observed decrease or increment of agglomeration by changing $\mathrm{pH}$. Finally, it should be noted that optimized $\mathrm{pH}$ value is different from one sample to another. For instance, suitable $\mathrm{pH}$ value for alumina, copper and graphite dispersed in water are around $8,9.5$ and 2 , respectively [30].

\subsubsection{Ultrasonic agitation:}

After preparation of nanofluids, agglomeration might occur over the time which results in fast sedimentation of nanoparticles due to enhancement of downward body force. Manson et al.[33] Investigated two different nanofluids; carbon black-water and silver-silicon oil and they utilized high energy of cavitation for breaking clusters among particles.

\section{Stability Mechanisms}

Stability which is the most crucial issue can be hampered by particle aggregation. Aggregation of nanoparticles is due to the sum of attractive and repulsive forces between particles. If attractive forces prevail over repulsive one then particle aggregate in clusters. Therefore enhancement of repulsive forces over attractive forces can prevent particle aggregation and ensure stability. Enhancement can be done by two mechanisms: electrostatic stabilization and steric stabilization. Here these two mechanisms are discussed in brief.

\subsection{Electrostatic stabilization}

Existence of an electric charge on the surfaces of particles is a major source of kinetic stability. Electrostatic stabilization occurs by adsorption of ions to the electrophilic metal surface (Figure6). Adsorption creates an electrical double/multi-layer which results in a Columbic repulsion force between the nanoclusters. Electrostatic stabilization is a $\mathrm{pH}$ sensitive method and of limited use.

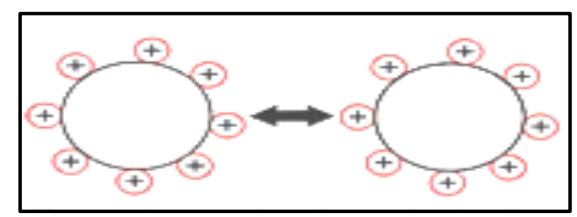

Figure6: Electrostatically stable nanoparticle

\subsection{Steric stabilization}

Steric stabilization of nanoparticles is achieved by attaching (grafting or chemisorption) macromolecules such as polymers or surfactants to the surfaces of the particles (Figure7). The stabilization is due to the large adsorbents which provide steric barrier to prevent particles coming close to each other. For example, stability of graphite nanofluids is due the protective role of PVP as it prevents the agglomeration of nanoparticles due to steric effect [18].

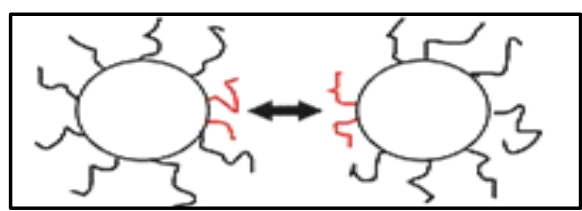

Figure7: Sterically stabilized nanoparticle 


\section{Conclusion and Remarks:}

In this review article an attempt has made to cover all the important investigations performed on the preparation and stability of nanofluids. We have come to know that two-step preparation method is accepted by the major portion of researchers as it is simple and economic. Further, we have found the steps involved in the stabilization process of these fluids. Additionally, mechanisms corresponding to stability of such fluids are also discussed.

Although there are some positive outcomes of nanofluid,but the following issues should receive greater attention for their proper utilization.

- Fault free economic production technique is the most important thing for the commercialization of nanofluids.

- The shape of nanoparticles is very important for their properties; therefore a new preparation method with controllable microstructure will be an interesting research topic.

- Long term of stability and stability after a long working cycle should paid intense attention for both research and real life application.

- Development of suitable surfactants for better stability of nanofluids may be a topic of interest.

References

[1] R. Taylor, S. Coulombe, T. Otanicar, P. Phelan, A. Gunawan, W. Lv, G. Rosengarten, R.Prasher, H.Tyagi, “Small particles, big impacts: A review of the diverse applications of nanofluids",J. Appl. Phys. 113, 011301 (2013), (http://dx.doi.org/10.1063/1.4754271).

[2] H. Akoh, Y.Tsukasaki, S.Yatsuya, and A.Tasaki, "Magnetic properties of ferromagnetic ultrafine particlesprepared by vacuum evaporation on running oil substrate." Journal of Crystal Growth, 45, 495-500, 1978.

[3] J.A.Eastman, U.S.Choi, S.Li, L.J.Thompson, S.Lee, "Enhanced thermal conductivity through the development of nanofluids", Materials Research Society Symposium-Proceedings, Materials research Society, Pittsburgh, PA, USA, Boston, MA, USA, vol.457:pp.3-11, 1997.

[4] H.T.Zhu, Y.S.Yin, A, "A novel one-step chemical method preparation of copper nanofluids", Journal of Colloid and Interface Science, vol.227, no.1, pp.100-130, 2004

[5] C-H. Lo, T-T. Tsung, L-C. Chen, C.-H. Su and H.-M.Lin, "Fabrication of Copper Oxide Nanofluid Using Submerged Arc Nanoparticle Synthesis System (SANSS)".Journal of Nanoparticle Research 7: 313-320, 2005.

[6] S. Lee, S. U.S. Choi, S. Li, and J. A. Eastman, "Measuring thermal conductivity of fluids containing oxide nanoparticles," Journal of Heat Transfer, vol. 121, no. 2, pp. 280-289, 1999.

[7] X. Wang, X. Xu, and S. U. S. Choi, "Thermal Conductivity of Nanoparticle-Fluid Mixture," Journal of Thermophysics and Heat Transfer 13: 474-480, 1999.

[8] S. M. S. Murshed, K. C. Leong, and C. Yang, "Enhanced Thermal Conductivity of TiO 2 -Water Based Nanofluids", International Journal of Thermal Sciences 44: 367-373, 2005.

[9] Y. Xuan and Q. Li, "Heat transfer enhancement of nanofluids," International Journal of Heat and Fluid Flow, vol. 21, no. 1, pp. 58$64,2000$.

[10] K.Kwak, C.Kim, Kor.-Austr. Rheol. J., 17, 35, 2005.

[11] J. A. Eastman, S. U. S. Choi, S. Li, W. Yu, and L. J. Thompson, "Anomalously increased effective thermal conductivities of ethylene glycol-based nanofluids containing copper nanoparticles,” Applied Physics Letters, vol. 78, no. 6, pp. 718-720, 2001.

[12] X. Feng, H. Ma, S. Huang et al., Aqueous-organic phase-transfer of highly stable gold, silver, and platinum nanoparticles and new route for fabrication of gold nanofilms at the oil/water interface and on solid supports, Journal of Physical Chemistry B, vol. 110, no. 25, pp. 12311-12317, 2006.

[13] W. Yu, H. Xie, L. Chen, and Y. Li, "Enhancement of thermal conductivity of kerosene-based $\mathrm{Fe}_{3} \mathrm{O}_{4}$ nanofluids prepared via phasetransfer method," Colloids and Surfaces A, vol. 355, no. 1-3, pp. 109-113, 2010.

[14] X. Wei and L.Wang, "Synthesis and thermal conductivity of microfluidic copper nanofluids," Particuology, vol. 8, no. 3, pp. 262$271,2010$.

[15] H. T. Zhu, C. Y. Zhang, Y. M. Tang, and J. X. Wang, "Novel synthesis and thermal conductivity of CuO nanofluid," Journal of Physical Chemistry C, vol. 111, no. 4, pp. 1646-1650, 2007.

[16] H. J. Kim, I. C. Bang, and J. Onoe, "Characteristic stability of bare Au-water nanofluids fabricated by pulsed laser ablation in liquids," Optics and Lasers in Engineering, vol. 47, no. 5, pp. 532-538, 2009.

[17] D. Zhu, X. Li, N. Wang, X. Wang, J. Gao, and H. Li, "Dispersion behavior and thermal conductivity characteristics of $\mathrm{Al}_{2} \mathrm{O}_{3}-\mathrm{H}_{2} \mathrm{O}$ nanofluids," Current Applied Physics, vol. 9, no. 1, pp. 131-139, 2009.

[18] H. Zhu, C. Zhang, Y. Tang, J. Wang, B. Ren, and Y. Yin, "Preparation and thermal conductivity of suspensions of graphite nanoparticles," Carbon, vol. 45, no. 1, pp. 226-228, 2007.

[19] A. K. Singh and V. S. Raykar, "Microwave synthesis of silver nanofluids with polyvinylpyrrolidone (PVP) and their transport properties," Colloid and Polymer Science, vol. 286, no. 14-15, pp. 1667-1673, 2008.

[20] Y. Hwang, J. K. Lee, C. H. Lee et al., "Stability and thermal conductivity characteristics of nanofluids," Thermochimica Acta, vol. 455, no. 1-2, pp. 70-74, 2007.

[21] X. Li, D. Zhu, and X. Wang, "Evaluation on dispersion behavior of the aqueous copper nanosuspensions," Journal of Colloid and Interface Science, vol. 310, no. 2, pp. 456-463, 2007.

[22] M. Chandrasekar, S. Suresh, A. Chandra Bose, "Experimental investigations and theoretical determination of thermal conductivity and viscosity of $\mathrm{Al}_{2} \mathrm{O}_{3}$ /water nanofluid," Exp. Therm. Fluid Sci. 34 (2), pp.210-216, 2010.

[23] Y. Hwang, J-K. Lee, J-K. Lee, Y-M. Jeong, S-i.Cheong, Y-C. Ahn, S.H. Kim, "Production and dispersion stability of nanoparticles in nanofluids," PowderTechnol. 186 (2), pp.145-153, 2008.

[24] X.F. Li, D.S. Zhu, X.J. Wang, N. Wang, J.W. Gao, H. Li, Thermal conductivity enhancement dependent pH and chemical surfactant for $\mathrm{Cu}-\mathrm{H}_{2} \mathrm{O}$ nanofluids, Thermochim. Acta 469 (1-2),pp. 98-103,2008. 
[25] M.N. Pantzali, A.A. Mouza, S.V. Paras, "Investigating the efficiency of nanofluids as coolants in plate heat exchangers (PHE)," Chem. Eng. Sci. 64 (14), pp.3290-3300, 2009.

[26] I. Madni, C.-Y. Hwang, S.-D. Park, Y.-H. Choa, H.-T. Kim, Mixed surfactant system for stable suspension of multiwalled carbon nanotubes, Colloids Surface A: Physicochem. Eng. Aspects358 (1-3), pp.101-107, 2010.

[27] W. Yu, H. Xie, "A Review on Nanofluids: Preparation, Stability Mechanisms, and Applications," Journal of Nanomaterials, vol2012, pp.1-17, 2012.

[28] X. Yang and Z. H. Liu, "A kind of nanofluid consisting of surface-functionalized nanoparticles, "Nanoscale Research Letters, vol. 5, no. 8, pp. 1324-1328, 2010.

[29] L. Chen and H. Xie, "Surfactant-free nanofluids containing double- and single-walled carbon nanotubes functionalized by a wetmechanochemical reaction," Thermochimica Acta, vol. 497, no. 1-2, pp. 67-71, 2010.

[30] Q. Yu, Y. J. Kim, and H. Ma, "Nanofluids with plasma treated diamond nanoparticles," Applied Physics Letters, vol. 92, no. 10, Article ID 103111, 2008.

[30] H. Xie, H. Lee, W. Youn, M. Choi, "Nanofluids containing multiwalled carbon nanotubes and their enhanced thermal conductivities," J. Appl. Phys. 94 (8), pp.4967-4971, 2003.

[31] D. Wen, G. Lin, S. Vafaei, and K. Zhang, "Review of nanofluids for heat transfer applications, ”Particuology, vol. 7, no. 2, pp. 141$150,2009$.

[32] Y. Fovet, J. Y. Gal, and F. Toumelin-Chemla, "Influence of pH and fluoride concentration on titanium passivating layer: stability of titanium dioxide," Talanta, vol. 53, no. 5, pp. 1053-1063, 2001.

[33] X. J. Wang and X. F. Li, "Influence of pH on nanofluids' viscosity and thermal conductivity," Chinese Physics Letters, vol. 26, no. 5, Article ID 056601, 2009.

[34] B.R. Munson, D.F. Young, T.H. Okiishi, Fundamentals of Fluid Mechanics, John Wiley \& Sons Inc., 1998.

[35] D. W. Oh, A. Jain, J. K. Eaton, K. E. Goodson and J. S. Lee, "Thermal Conductivity Measurement and Sedimentation Detection of Aluminum Oxide Nanofluids by Using the3 $\mathrm{\omega Method,"} \mathrm{International} \mathrm{Journal} \mathrm{of} \mathrm{Heat} \mathrm{and} \mathrm{Fluid} \mathrm{Flow,} \mathrm{ISSN:} \mathrm{0142-727X,} \mathrm{vol.} \mathrm{29,} \mathrm{no.}$ 5, pp. 1456-1461, 2008.

[36] X. Wei, H. Zhu, T. Kong, L. Wang, "Synthesis and thermal conductivity of $\mathrm{Cu}_{2} \mathrm{O}$ nanofluids," International Journal of Heat and Mass Transfer, vol. 52, no.19-20, pp.4371-4374, 2009.

[37] D. Wu, H. Zhu, L. Wang, L. Liua, Curr. NanoSci, vol.5, pp.103-112, 2009.

[38] H. Zhu, D. Han, Z. Meng, D. Wu, C. Zhang, "Preparation and thermal conductivity of CuO nanofluid via a wet chemical method." Nanoscale Research Letters, vol.6, no.1, p.181, 2011

[39] P. Razi, M.A. Akhavan-Behabadi, M. Saeedinia,"Pressure drop and thermal characteristics of CuO-base oil nanofluid laminar flow in flattened tubes under constant heat flux," International Communications in Heat and Mass Transfer,vol.38,pp.964-971,2011.

[40] P. Keblinski, J. A. Eastman, D. G. Cahill, "Nanofluids for Thermal Transport,” Materials Today, June, pp.36-44, 2005.

[41] M-S. Liu, M. C-C. Lin, I-T.Huang, C.-C.Wang, "Enhancement of thermal conductivity with carbon nanotube for nanofluids."vol.32, no.9, pp.1202-1210, 2005. 